\section{Steffen K. HerrmanN}

\section{Drei Pathologien der Anerkennung}

\author{
Grundlagen einer kritischen Gesellschaftstheorie \\ nach Rousseau, Hegel und Marx
}

Das Unternehmen einer kritischen Gesellschaftstheorie entspringt einer Umbruchszeit, die Mitte des I 8. Jahrhunderts durch den Geist der Aufklärung und die industrielle Revolution ausgelöst wurde. Vorhaben der Aufklärung war es, die Individuen in die Lage zu versetzen, alle sozialen, gesellschaftlichen und politischen Verhältnisse zum Gegenstand der Reflexion zu machen und diese nur dann zu akzeptieren, wenn sie sich als vernünftig erweisen. Die Individuen sollten so in die Lage versetzt werden, all jene Herrschaftsverhältnisse in Frage zu stellen und abzuschütteln, die nicht als Ausdruck ihrer Selbstbestimmung verstanden werden könnten. Im gleichen geschichtlichen Moment jedoch, wo die Aufklärung den Menschen aus seinen Ketten befreien wollte, trat mit der industriellen Revolution eine neue gesellschaftliche Macht auf, die bald weite Teile der Bevölkerung dazu zwang, sich in unsicheren Lohnarbeitsverhältnissen gegen schlechte Bezahlung zu verdingen. Die damit verbundene Verarmung äußerte sich nicht nur in materiellem Elend, sondern auch in in der sozialen Desintegration der Individuen, die in der Folge unter dem Stichwort der "sozialen Frage" diskutiert wurde (Donzelot I984: Castel 2000).

Es ist eben diese Gemengelage aus emanzipatorischen Ansprüchen und materiellen Abhängigkeiten, in welcher die kritische Gesellschaftstheorie als eigene Disziplin erblickt das Licht der Welt. Mit den Werken von Jean-Jacques Rousseau, Georg Wilhelm Friedrich Hegel und Karl Marx erscheinen dabei in einem Abstand von knapp einhundert Jahren einschlägige Gründungstexte. Allen drei Autoren ist dabei das Vorhaben gemein, sich kritisch-reflexiv mit den Wandlungen ihrer jeweiligen Zeit auseinandersetzen zu wollen: Rousseau im absolutistischen Frankreich, Hegel im agrarischen Preußen und Marx im industrialisierten England. Trotz der unterschiedlichen historischen und nationalen Kontexte gibt es dabei ein verbindendes Moment zwischen den drei Autoren. Sie alle versuchen nämlich, die sozialen Pathologien ihrer Zeit als Pathologien der Anerkennung zu verstehen. Soziale Desintegration, so ihre Überzeugung, darf nicht allein vor dem Hintergrund materieller Ungleichheit verstanden werden, sondern muss selbst noch einmal als Ausdruck verweigerter Teilhabe gedeutet werden. Die Arbeiten von Rousseau, Hegel und Marx zeichnen sich nun dadurch aus, diese grundlegende Einsicht auf ganz unterschiedliche Art und Weise zu einer je spezifischen Pathologiediagnose ausgeweitet zu haben: Während Rousseau das Phänomen der Anerkennungsbesessenheit in den Mittelpunkt seiner Überlegungen rückt, ist es bei Hegel das Phänomen der Anerkennungsabhängigkeit und bei Marx das Phänomen der Anerkennungsvergessenheit.

Ich will es mir im Folgenden nun zur Aufgabe machen, die Pathologiediagnosen von Rousseau, Hegel und Marx nacheinander zu rekonstruieren und ihre gegenwärtige Bedeutung aufzuzeigen. Dabei werde ich in meiner Rekonstruktionen der drei Autoren jeweils in drei Schritten vorgehen: In einem ersten Schritt werde ich die normativen Grundlagen der jeweiligen Anerkennungstheorie herausarbeiten und in Erinnerung rufen, um dann vor diesem Hintergrund in einem zweiten Schritt die je spezifische Pathologiediagnose entwickeln zu können. In einem dritten Schritt wird es mir dann darum gehen, zu zeigen, wie die jeweiligen Pathologiediagnosen von der Sozialtheorie des 20 . Jahrhunderts aufgenommen worden sind, um so die Gegenwärtigkeit der von Rousseau, Hegel und Marx diagnostizierten Probleme deutlich zu machen und zu zeigen, wie ihre Ansätze auch heute noch als Grundlagen einer kritischen Gesellschaftstheorie fungieren können.

\section{Rousseau: Die Besessenheit von Anerkennung}

Noch bevor der Begriff der Anerkennung mit Fichte und Hegel zu einem Grundbegriff der praktischen Philosophie werden sollte, spielt er in der Philosophie von Rousseau eine zentrale Rolle. Rousseaus Überlegungen nehmen dabei eine besondere Stellung ein, weil sie sich von Anfang an mit den ambivalenten Effekten des intersubjektiven Anerkennens auseinandersetzen. Die Anerkennung von Anderen, so zeigt uns Rousseau, ist Segen und Úbel zugleich: Einerseits bildet sie eine unhintergehbare Voraussetzung für gelingende Selbstverwirklichung, andererseits geht mit ihr die Gefahr der Entfremdung des menschlichen Lebens einher. Da Rousseau in seinen kulturkritischen Schriften vor allem letztere in den Vordergrund gerückt hat, schien es vielen Rousseau-Interpreten lange Zeit so, als wäre Rousseau allein ein Anerkennungskritiker. Für eine Korrektur dieses Bildes haben in jüngster Zeit vor allem die Arbeiten von Frederick Neuhouser gesorgt, der gezeigt hat, dass Rousseau vor allem in seinen pädagogischen und politischen Schriften eine positive Theorie der Anerkennung entwirft (Neuhouser 2008: 20I2). Im Anschluss an diese Überlegungen werde ich im Folgenden zunächst ausgehend von Rousseaus Konzept der amour propre den normativen Status der Anerkennung herausarbeiten (i), um mich dann in einem zweiten Schritt jener Pathologie zuwenden, die für Rousseau im Zentrum der Moderne steht: die 
Anerkennungsbesessenheit (ii). Im dritten Schritt werde ich dann nach der Gegenwart von Rousseaus Pathologiediagnose fragen und prüfen inwiefern wir es hier mit einer spezifischen Pathologie der Moderne zu tun haben.

(i) Um die Grundlagen von Rousseaus Anerkennungstheorie freizulegen, muss man sein Werk gleichsam von hinten nach vorne lesen. Vom normativen Standpunkt aus betrachtet, geht der 1762 erstmals publizierte Emile dem sieben Jahre früher erschienenen Diskurs über die Ungleichbeit nämlich voraus. Erst vor dem Hintergrund der dort entwickelten normativen Überlegungen lassen sich die im Diskurs skizzierten gesellschaftlichen Fehlentwicklungen nämlich als Fehlentwicklungen verstehen. Liest man den Diskurs über die Ungleichbeit ohne diesen Hintergrund, läuft man Gefahr, den von Rousseau skizzierten Prozess entweder so zu deuten, als wolle er für eine Rückkehr zu einem Zustand vor jeder Vergesellschaftung zurückkehren oder als wolle er sagen, dass unser modernes Leben notwendig entfremdet sei. Weder das eine noch das andere ist jedoch der Fall. Rousseaus zivilisationskritische Überlegungen aus dem zweiten Diskurs müssen vielmehr so verstanden werden, dass sie uns gesellschaftliche Fehlentwicklungen vor Augen führen, die aus einem falschen Umgang mit der menschlichen Natur herrühren (Dent I988).

Wie die menschliche Natur beschaffen ist und was es bedeutet ihr gerecht zu werden, das zeigt uns Rousseau in seinem großen Erziehungsroman Emile. Der Emile gliedert sich dabei in fünf Büchern, die jeweils eine bestimmte Entwicklungsphase des Zöglings behandeln. Für die Rekonstruktion von Rousseaus Anerkennungstheorie ist vor allem die vierte Entwicklungsstufe von Bedeutung, weil hier der eigentliche Eintritt des Menschen in die Gesellschaft stattfindet. Die zentrale Stellung dieser Entwicklungsphase macht Rousseau dadurch deutlich, dass er von einer nun stattfindenden "zweiten Geburt « des Menschen spricht (Rousseau I 998: 2 I I ). Im Mittelpunkt dieser zweiten Geburt stehen vor allem zwei Leidenschaften: Die Selbstliebe (amour de soi-meme) und die Eigenliebe (amour propre) (ebd.: 2I2). Erstere zielt dabei auf unsere Selbsterhaltung, denn würden wir uns selbst nicht lieben, hätten wir kein Interesse daran, uns am Leben zu erhalten, lieben wir uns aber selbst, wird uns unsere Erhaltung und alle materiellen Güter, die dazu beitragen, wichtig. Anders verhält es sich mit der Eigenliebe: Sie zielt nicht auf ein materielles, sondern auf ein symbolisches Gut: die Anerkennung von Anderen. Für die Eigenliebe sind daher die Meinungen und Überzeugungen, die Andere von uns hegen, wesentlich.

Warum aber sollten die Meinungen und Überzeugungen, die andere von uns haben, für uns wesentlich sein? Zeichnet es uns als Menschen nicht gerade aus, auf die Meinung von anderen $\mathrm{zu}$ spfeifen ihr unabhängig machen zu können? Rousseaus Antwort auf diese Frage lautet, dass wir im Einzelfall zwar durchaus auf die Bestätigung durch Andere verzichten können, ganz ohne sie auszukommen uns jedoch nicht möglich ist. Im Diskurs über die Ungleichbeit formuliert Rousseau daher, dass der Mensch erst durch die Wertschätzung von Anderen dazu in der Lage sei, ein "Gefühl seiner eigenen Existenz" (Rousseau 2008: 269) zu erlangen. Was nun ist aber damit gemeint? Hinter dieser Formulierung verbirgt sich der Gedanke, dass ein Individuum eine gelungene Selbstbeziehung nur durch die Beziehung zu Anderen aufbauen kann. Wir können uns das an folgendem Beispiel deutlich machen: Ein Schriftsteller, der sich selbst für einen außergewöhnlichen Romanautor hält, wird diese Überzeugung nur dann aufrechterhalten können, wenn sie durch Andere bestätigt wird. Erst wenn diese ihm die Bestätigung geben, dass es sich bei dem von ihm geschaffenen Werk um etwas Außergewöhnliches handelt, kann er sich sicher sein, dass er ein stimmiges Selbstbild von sich hat. Erhält er dagegen keine solche Bestätigung von außen, wird es ihm auf Dauer nicht möglich sein, seine Überzeugung aufrechtzuerhalten. Zwar kann er sich als ein »verkanntes Genie « stilisieren, je länger er jedoch dazu gezwungen ist, in dieser Haltung zu verharren und sein Selbstbild gegen die Gesellschaft zu behaupten, umso mehr wird er in Gefahr geraten, den Standpunkt der Verrücktheit einzunehmen (Neuhouser 20I2: 54). Wenn Rousseau die Eigenliebe daher als die Quelle des »Gefühls der eigenen Existenz « bezeichnet, dann macht er deutlich, dass wir einen positiven Bezug zu unserer eigenen Existenz nur dann aufbauen können, wenn signifikante Andere das Bild, das wir uns von uns selbst machen, bestätigen und befürworten.

Zwei grundsätzlich unterschiedliche Formen der Liebe werden also bei Rousseau unterschieden: Die amour de soi-même, welche die Reproduktion unseres physischen Lebens betrifft auf der einen und die amour-propre, welche auf die Reproduktion unseres sozialen Lebens zielt, auf der anderen Seite. Im Zuge seiner Überlegungen zur Entwicklung der geschlechtlichen Liebe versucht Rousseau zu zeigen, dass beide nicht einfach unabhängig voneinander existieren, sondern dass sich die amour-propre beim Menschen aufgrund seiner besonderen geistigen Fähigkeiten aus dem Naturtrieb heraus entwickelt: Das liegt daran, dass der Mensch mit der Fähigkeit zur Freiheit begabt ist, was zur Folge hat, dass er seine Liebesobjekte nicht instinkthaft vorfindet, sondern sie wählen muss. Und diese Wahl ist bei ihm keine blinde Wahl, denn sie basiert aufgrund seiner Fähigkeiten zum Vergleich auf einem qualifizierenden Urteil. Das Liebesobjekt zeichnet sich für Rousseau so von Anbeginn an durch eine gewisse Vortrefflichkeit aus. Diese Einsicht gilt jedoch sowohl für das Subjekt als auch für das Objekt der Liebe: "Gibt man jemandem den Vorzug, so will man auch vorgezogen werden: die Liebe muss gegenseitig sein « (Rousseau I 998: 2 I4). Was an dieser Stelle deutlich 
wird, ist, dass wir für Rousseau in der geschlechtlichen Beziehung immer auch die Tatsache genießen, von einer anderen Person für Vortrefflich gehalten zu werden. Demzufolge erwächst aus der geschlechtlichen Liebe stets eine zweifache Befriedigung: die snatürliche`Befriedigung des Geschlechtstriebs und die ssoziale 'Befriedigung, als Geschlechtspartner anerkannt und bestätigt worden zu sein. Und wenn Rousseau letzteres als ein "süßes Gefühl« (ebd.) bezeichnet, dann scheint er damit im Blick zu haben, dass die Anerkennung durch andere eine Befriedigung in sich darstellt, da sie einen positiven Bezug zur eigenen Existenz ermöglicht In der geschlechtlichen Liebe findet so der entscheidende Übergang von der amour de soi-même zur amour-propre statt. Fortan wird der Mensch nicht nur nach seiner Befriedigung als Naturwesen, sondern auch als Gesellschaftswesen suchen.

(ii) Nachdem im ersten Schritt deutlich geworden ist, welche Rolle die amour-propre im Leben des Subjekts spielt, will ich mich nun im zweiten Schritt den mit ihr verbundenen Gefahren zuwenden. Rousseau attestiert dem modernen Subjekt eine umfassende Anerkennungsbesessenheit, für die er im Emile drei psychologische Ursachen anführt: Entscheidend ist erstens, dass mit dem Begehren nach Liebe eine Abhängigkeitsbeziehung im Leben des Subjekts auftaucht. Durch sein Verlangen nach Liebe gerät der Mensch außer sich: er betrachtet sich mit den Augen von Anderen. Dieses Außer-sich-sein wird für Rousseau nun dadurch zum Extrem gesteigert, dass der Einzelne nicht nur von einer anderen Person geliebt werden möchte, sondern von allen Anderen. Er hält diesbezüglich fest: „Wer fühlt, wie süß es ist, geliebt zu werden, möchte von aller Welt geliebt werden « (Rousseau I 998: 2 I4). Zweitens setzt das universale Verlangen nach Liebe die Individuen in ein Wettbewerbsverhältnis, in dem sie mit anderen um die Anerkennung von Dritten buhlen. Das Verlangen nach Anerkennung führt so unweigerlich in einen Kampf um Anerkennung, in dem ein jeder versucht, den jeweils anderen zu übertrumpfen und so in einer bestimmten Hinsicht als der jeweils Beste anerkannt zu werden. Das wiederum bedeutet, dass man sich Anderen gegenüber als überlegen erweisen muss: "Daher dic ersten Blicke auf seinesgleichen, daher die ersten Vergleiche mit ihnen, daher der Wetteifer, die Rivalität und die Eifersucht.« (Ebd.) Drittens hat der Kampf um Anerkennung für Rousseau zur Folge, dass sich das Feld der Anerkennungsverhältnisse nahezu ins Unendliche ausdehnt. Das Streben nach nach Überlegenheit kann alle Bereiche des Lebens infizieren und diese für die Zwecke der Selbstdarstellung vereinnahmen. Sobald es etwa zum Brauch wird, das eigene Prestige auch in Dingen reflektiert zu sehen, können sogar die für das Leben unmittelbar relevanten Gebrauchsgüter in den Kampf um Anerkennung einbezogen und zu Prestigegütern werden. Die Individuen entwickeln so letztlich einen monomanischen Blick auf die Welt: Nicht nur gilt ihnen alles und jedes als Gegenstand der Anerkennung, vielmehr hat für sie jede Interaktion mit Anderen ausschließlich den Zweck, die eigene Überlegenheit zur Schau zu stellen. Anders gesagt: Die Abhängigkeit von der Anerkennung von Anderen führt für Rousseau in eine umfassende Anerkennungsbesessenheit.

Diese Besessenheit gilt Rousseau nun als Grund für eine Reihe von sozialen Pathologien, denen er sich ausführlich in seinem Diskurs über die Ungleichbeit widmet. Er skizziert hier mittels eines genealogischen Verfahrens einen fiktiven Entwicklungsprozess moderner Gesellschaften, der von einem heilen Naturzustand über die Epochen der ersten und zweiten Revolution hin zur Entstehung der bürgerlichen Gesellschaft führt (Neuhouser 20I4). Entgegen der Erwartungshaltung seiner damaligen Leserschaft hat Rousseau diesen Prozess dabei nicht als Fortschritts-, sondern vielmehr als Verfallsprozess konzipiert, in welchem sich die Menschen immer weiter in ihrer Abhängigkeit von der Anerkennung von Anderen verstricken. Drei Effekte der Anerkennungsbesessenheit hebt er dabei hervor:

Da die eigene soziale Position ein stellungsrelatives Gut ist, das sich im Verhältnis zur sozialen Position von Anderen bestimmt, misst sich das eigene Ansehen immer an dem Ansehen von Anderen. Anerkennung ist daher keine absolute Anerkennung, sondern immer nur Anerkennung »im Verhältnis zu «. Das hat zur Folge, dass dasjenige, was heute noch großes Ansehen beschert, schon morgen diesen Zweck nicht mehr erfüllen kann. Da Anerkennung weiterhin ein knappes Gut ist, verhalten sich das eigene Ansehen und das Ansehen von anderen komplementär zueinander: Der Zugewinn an Ansehen des Einen bedeutet für den Anderen einen Verlust. Die Relativität und Knappheit der Anerkennung haben für Rousseau zur Folge, dass die Menschen ein Interesse an der Ausbildung stabiler Ungleichheitsverhältnisse entwickeln. Rousseau denkt dabei in erster Linie an den Unterschied zwischen arm und reich, der für ihn durch den die Eigentumsordnung sichernden Gesellschaftsvertrag verstetigt wird: "Dies war oder muß der Ursprung der Gesellschaft und der Gesetze gewesen sein, die dem Schwachen neue Fesseln und dem Reichen neue Kräfte gaben « (Rousseau 2008: 219). Gleich nun, ob man Rousseaus Genealogie der modernen Gesellschaft teilt, bleibt doch ein Gedanke seiner Überlegungen wesentlich: Der Kampf um Anerkennung flößt den Individuen ein Interesse an der Etablierung und Verstetigung sozialer Ungleichheiten ein. Verbunden mit der Etablierung sozialer Ungleichheitsverhältnisse, spricht Rousseau davon, dass der Kampf um Anerkennung die sozialen Akteure statt zu Partnern zu machen, zu Gegnern macht. Die Relationalität der Anerkennung flößt den Einzelnen einen unmittelbaren Anreiz ein, sich wechselseitig Schaden zuzufügen: „Schließlich gibt der verzehrende Ehrgeiz, der Eifer, sein relatives Vermögen zu erhöhen [...], allen Menschen einen finsteren Hang ein, sich wechselseitig 
zu schaden « (ebd.: 209). Eine weitere Gefahr besteht darin, dass sich die Individuen von sich selbst entfremden. Und zwar in dem Sinne, dass sie sich nicht mehr um ihr tatsächliches Sein, sondern nur noch um ihren gesellschaftlichen Schein kümmern: "Sein und Scheinen wurden zwei völlig verschiedene Dinge, und aus diesem Unterschied gingen der aufsehenerheischende Pomp, die betrügerische List und alle Laster hervor, die zu ihrem Gefolge gehörten « (ebd.: 207). Weil es unter Umständen einfacher sein kann, Andere dazu zu bringen, uns als vortrefflich wahrzunehmen, statt wirklich vortrefflich zu werden, fördert der Kampf um Anerkennung Laster wie Täuschung, Heuchelei und Doppelzüngigkeit, die allesamt zu einem gestörten Selbstverhältnis führen, insofern die Bestätigung von Anderen hier einem Selbstbild gilt, in welchem sich das Individuum letztlich selbst gar nicht wiedererkennen kann.

Die Entstehung von sozialer Ungleichheit, die Förderung von Lastern, und die Entfremdung vom eigenen Selbst sind drei wichtige Konsequenzen, die für Rousseau aus der Anerkennungsbesessenheit moderner Gesellschaften folgen. Wie gesehen, führt er diese auf drei psychologische Dispositionen zurück: Das universelle Verlangen nach Liebe, das Streben nach Überlegenheit und das Streben danach, diese Überlegenheit in allen Dingen zum Ausdruck zu bringen. Alle drei Motive möchte ich nun abschließend prüfen, um beurteilen zu können, inwiefern wir heute noch berechtigt an das von Rousseau diagnostizierte Phänomen der Anerkennungsbesessenheit anknüpfen können.

(iii) Die erste Voraussetzung von Rousseaus Argumentation lautete, dass der moderne Mensch von der ganzen Welt geliebt werden will. Rousseau präsentiert uns als erste Ursache der Anerkennungsbesessenheit daher mehr oder weniger eine anthropologische Disposition. Es liegt in der Natur des Menschen, einen unersättlichen Hunger nach Anerkennung zu haben. Meiner Meinung nach lässt sich diese fragliche anthropologische Annahme Rousseaus konzeptionell folgendermaßen retten: Prinzipieil lassen sich drei Sorten von Anerkennungsbeziehungen unterscheiden - solche, in denen wir für jene Eigenschaften anerkannt werden, die wir mit allen anderen Menschen teilen, solche, die wir nur mit einigen teilen und mit anderen nicht, und solche, die uns von allen anderen unterscheiden. Wir können diesbezüglich von einer egalisierenden, einer partikularisierenden und einer individuierenden Anerkennung sprechen. Die von Rousseau skizzierte Gefahr eines Anerkennungswettkampfes taucht nun vor allem im Fall der individuierenden Anerkennung auf. Nur dort, wo es darum geht, sich von Anderen zu unterscheiden, birgt der Vergleich das Potential in einen Kampf um Anerkennung auszuarten. Was Rousseau psychologisch als ein umfassendes Verlangen nach Liebe deutet, können wir im Anschluss an Charles Taylor auch als ein spezifisches Kennzeichen der Moderne beschreiben: Deren Spezifikum besteht laut Taylor ja gerade in dem Zerfall tradierter Anerkennungsordnungen, in welchen die Einzelnen Wertschätzung im Zuge ihrer Standes-, Innungs- oder Familienzugehörigkeit erhalten. Moderne Anerkennungsordnungen zeichnen sich für Taylor dagegen dadurch aus, dass die Einzelnen hier als Einzelne zur Geltung kommen wollen und sollen (Taylor I 996). Das Signum der Moderne, so können wir sagen, besteht gerade im Anspruch auf Individualität - und eben deshalb kommt der individuierenden Anerkennung in der Moderne ein Stellenwert zu, den sie bis dato nicht besessen hat. Was Rousseau daher zunächst psychologisch zu erläutern versucht, können wir als das Resultat eines spezifisch gesellschaftlichen Modernisierungsprozesses verstehen.

Die zweite Prämisse von Rousseaus Argumentation lautete, dass das Verlangen nach Liebe mit einem Streben nach Überlegenheit verbunden ist. Ich denke wir können diese Prämisse Rousseaus besser verstehen, wenn wir uns über den spezifischen Charakter individuierender Anerkennung klar werden. Individuierende Anerkennung bedeutet, für Eigenschaften oder Fähigkeiten geschätzt zu werden, die uns von anderen in positiver Weise unterscheiden. Ihr liegt dabei ein kontrastives Moment zu Grunde, insofern sie Fähigkeiten, Leistungen oder Eigenschaften hervorhebt, die uns evaluativ von anderen unterscheiden. Eben dieses Differenzkriterium hat aber zur Folge, dass Gegenstand von individuierender Anerkennung zu sein bedeutet, in einer spezifischen Hinsicht als herausragend und damit als besser als Andere anerkannt zu werden (Neuhouser 201 2: $45 \mathrm{ff}$.). Das Streben nach Wertschätzung ist daher intrinsisch mit dem Versuch verbunden, Andere in bestimmten Hinsichten zu übertrumpfen. Das von Rousseau skizzierte Streben nach einer Vorrangstellung ist dann aber wiederum kein anthropologisches Grundmerkmal, sondern einfach ein Kennzeichen dessen, was individuierende Anerkennung konzeptionell ausmacht.

Als dritte Prämisse seiner Argumentation hatte Rousseau schließlich die Neigung ins Feld geführt, alles und jedes zum Gegenstand von Anerkennung zu machen. Fragen wir auch hier wieder nach den gesellschaftlichen Rahmungen dieser Disposition, dann zeigt sich, dass ein Kennzeichen unserer modernen Marktgesellschaften darin besteht, den Vergleich mit Anderen zum gesellschaftlichen Prinzip zu erheben. Von Seiten der Anbieter und der Produzenten korreliert der Markt dabei mit dem Wettbewerbsprinzip: Dieses besteht unter anderem darin, sich mit Anderen zu vergleichen und zu versuchen, in diesem Vergleich besser als diese abzuschneiden. Eben dieses Wettbewerbsprinzip hat im Zuge des Neoliberalismus eine umfassende gesellschaftliche Ausdehnung erfahren: Bildung, Gesundheit, Politik und Partnerwahl werden zunehmend wettbewerblich organisiert, was zur Folge hat, dass eine immer größere Anzahl an Relevanzkriterien bereitsteht, anhand derer sich Individuen scheinbar sobjektiv`zueinander ins Verhältnis setzen können. Die 
Möglichkeiten einander zu übertreffen und miteinander in Konkurrenz zu treten vervielfachen sich also durch die Ausdehnung des Marktprinzips auf vormals nicht wettbewerblich organisierte Bereiche zusehends. Der Marktgesellschaft wohnt daher die Tendenz inne, immer neue Anerkennungsmärkte zu erschließen (Rosa 2012: $324 \mathrm{ff}$.).

Neben dieses quantitative Moment gesellt sich zugleich aber auch noch ein qualitatives Moment: Während der Übergang vom Feudalismus zur Aufklärung als jener Übergang beschrieben werden kann, in welchem soziale Positionen nicht mehr traditionell vorgegeben sind, sondern individuell über Leistung erworben werden können, verändert sich die mit dem Leistungsprinzip einhergehende Ordnung im Neoliberalismus erneut: Beschäftigungs- und Familienverhältnissee sowie politische oder religiöse Orientierung sind nicht mehr auf lebenslange Stabilität hin angelegt, sondern werden in Abhängigkeit von der jeweiligen Performanz ständig neu geordnet. Diese Umstellung der Anerkennungsordnung von sozialer Lage hin zu sozialer Performanz hat zur Folge, dass die Individuen im Neoliberalismus zum Gegenstand kontinuierlicher Evaluationen und Bewertungen werden, in denen sie ihre Anerkennungswürdigkeit ständig unter Beweis stellen müssen (Rosa 201 3: $83 \mathrm{ff}$.). Ebenso wie durch die quantitative Ausdehnung des Wettbewerbs vervielfältigen sich auch durch die qualitative Steigerung des Wettbewerbs die äußeren Notwendigkeiten, in einen Anerkennungskampf treten zu müssen.

Die individuellen psychologischen Dispositionen, die Rousseau als Ursachen von Anerkennungsbesessenheit anführt, können also nicht ohne bestimmte gesellschaftliche Hintergrundbedingungen verstanden werden. Die Bedeutung, welcher individuierender Anerkennung in der Moderne zukommt, ihre innere Beschaffenheit und ihre qualitative und quantitative Ausdehnung können als jene gesellschaftlichen Voraussetzungen gelten, welche das Phänomen der Anerkennungsbesessenheit überhaupt erst möglich machen. Die Wiederaufnahme von Rousseaus Kritik der Anerkennungsbesessenheit sollte heute daher weniger in der Forderung nach einer Transformation der Dispositionen der Individuen bestehen, als vielmehr in der Fortführung der Kritik der gesellschaftlichen Anerkennungsverhältnisse.

\section{Hegel: Die Abhängigkeit von Anerkennung}

Während Rousseau die normativen Grundlagen seiner Anerkennungstheorie aus der Ontogenese des Subjekts gewinnt, möchte Hegel diese seiner Philosophie des Geistes entnehmen. Das wohl prominenteste Theoriestück seiner Geistphilosophie bilden dabei die Überlegungen zum Verhältnis von Herrschaft und Knechtschaft aus der Phänomenologie des Geistes (Kojève I 975). Die dort auf wenigen Seiten entfaltete
Auseinandersetzung hat geistesgeschichtlich eine solch enorme Strahlkraft entfaltet, dass sie überall dort, wo es intersubjektive Konflikt- und Ungleichheitsverhältnisse zu denken galt, als Blaupause gedient hat: Neben Marx waren es dabei vor allem Autoren wie Fanon, Sartre oder Lacan, die das Hegelsche Motiv des Kampfes um Anerkennung zu einem festen Bestandteil ihrer Sozialtheorie haben werden lassen (Kuch 2012). Durchweg war es dabei das Anliegen dieser Autoren, deutlich zu machen, dass sich hinter dem Konflikt zwischen den Individuen eine wechselseitige Angewiesenheit verbirgt. Streit und Entzweiung, so die mit dem Kampf um Anerkennung verbundene These, sind nicht Ausdruck einer feindseligen und asozialen Natur des Menschen, sondern vielmehr gerade Ausdruck seiner existenziellen Angewiesenheit auf Andere. Es ist eben dieser Gedanke, dessen normative Grundlagen ich im Folgenden in einem ersten Schritt freilegen möchte (i), bevor ich mich dann in einem zweiten Schritt jener Pathologie zuwende, die wir mit Hegel als Anerkennungsabhängigkeit beschreiben können (ii). Im letzten Schritt meiner Rekonstruktion möchte ich dann zeigen, wo diese Anerkennungsabhängigkeit heute ihre gesellschaftliche Realität erlangt hat (iii).

(i) Den Ausgangspunkt von Hegels Phänomenologie des Geistes bildet die Überzeugung, dass sich dessen logische und geschichtliche Entwicklung mit Hilfe der dialektischen Methode rekonstruieren lässt, in welcher der philosophische Begriff sein jeweils Anderes aus sich hervorbringt, um dann in einem höheren Begriff aufgehoben zu werden. Im Zuge dieser Entwicklungslogik taucht das Konzept der Anerkennung an eben jener Stelle auf, an welcher sich Hegel dem Begriff des Selbstbewusstseins zuwendet. Hegels These lautet dabei, dass sich der Begriff des Selbstbewusstseins logisch nur im Ausgang vom Konzept der Anerkennung sinnvoll explizieren lässt. Werkgeschichtlich findet sich diese Idee sowohl sowohl in der Phänomenologie des Geistes (1 806) als auch in der Enzyklopädie der Wissenschaften ( I 832) ausgearbeitet. Auch wenn die Überlegungen aus der Enzyklopädie zum Teil prägnanter sind, möchte ich mich im Folgenden der Phänomenologie zuwenden, da sich Hegel hier ausführlich dem Verhältnis von Herrschaft und Knechtschaft widmet und sich dabei als ein exzellenter Pathognostiker zeigen wird. Bevor wir jedoch zu Hegels Pathologiediagnose kommen, gilt es zunächst die normativen Grundlagen seiner Überlegungen zu klären.

Ausgangspunkt von Hegels logisch-begrifflicher Grundlegung des Anerkennungsprinzips ist der Begriff des Selbstbewusstseins, den Hegel mit Hilfe der Formel "Ich bin Ich" (Hegel I989: I 38) wiedergibt. Diese soll die Überzeugung des Selbstbewusstseins zum Ausdruck bringen, nur das zu sein, was es sein will. Es ist daher gerade die Fähigkeit zur Selbstbestimmung, durch die sich das Selbstbewusstsein für Hegel auszeichnet. Diese Fähigkeit ist jedoch so lange abstrakt und leer, wie sie sich nur 
in den eigenen Überzeugungen manifestiert. Solange das Selbstbewusstsein sich nur im Raum seines eigenen Denkens bewegt, kann es keine Gewissheit davon erlangen, ob die Überzeugungen, die es von sich hegt, auch wirklich zutreffen. Im Anschluss an Hegel können wir davon sprechen, dass das Selbstbewusstsein an einem Mangel an Gewissheit leidet (Herrmann 20I 2: $73 \mathrm{ff}$.). Dieser lässt sich nur dadurch aufheben, dass das Selbstbewusstsein praktisch in der Welt tätig wird und versucht seine Überzeugungen von sich zu vergegenständlichen. Hegel spricht davon, dass sich das Selbstbewusstsein die "Gestalt des Seins" (Hegel I989: I 38 ) verleihen muss. Der Begriff des Selbstbewusstseins bringt so notwendig sein Anderes aus sich hervor: den Begriff der Welt. Ohne diesen, so Hegels Überzeugung, lässt sich der Begriff des Selbstbewusstseins nicht angemessen fassen. Selbstbewusstsein und Welt schließen sich ein. Zwei grundlegend unterschiedliche Arten des Weltverhältnisses unterscheidet Hegel im Folgenden:

Zunächst die Praxis der Vergegenständlichung durch Arbeit. Sofern der Arbeitende durch den Prozess des Herstellens der Materie eine von ihm gewählte Form aufprägen kann, vermag ihm sein Selbstbewusstsein im Objekt als etwas Gegenständliches gegenüberzutreten. Die Tätigkeit des Herstellens ist daher nicht nur einfach eine Verausgabung von Arbeitskraft, sondern zugleich auch eine Realisierung von Selbstbewusstsein. Denken wir diesbezüglich etwa wieder an den bereits bei Rousseau erwähnten Schriftsteller, der einen Roman schreibt und anschließend an diesem seine kreativen Fähigkeiten anschauen kann, dann wird klar, inwiefern das Arbeiten eine Verwirklichung von Selbstbewusstsein bedeutet: Im Gegenstand sind diese Fähigkeiten materialisiert, wodurch sie nicht mehr nur ideell in Gedanken, sondern objektiv in der Welt existieren. Für Hegel ist das Arbeiten jedoch noch eine unvollkommene Form der Realisierung von Selbstbewusstsein. Das liegt daran, dass die Objektivität, die der Schriftsteller seinem Selbstverständnis durch die Niederschrift des Romans gegeben hat, nicht an sich, sondern nur für sich existiert. Zwar ist es ihm gelungen, sein Selbstverständnis zu vergegenständlichen, letztlich findet er in diesem Gegenstand aber nur das wieder, das er in ihn hineinlegt hat - nämlich seine Überzeugung, dass es sich um das Werk einer kreativen Persönlichkeit handelt. Der Praxis der Vergegenständlichung mangelt es also an Objektivität. Diese kann für Hegel nur dadurch erreicht werden, dass zur Praxis der Vergegenständlichung eine andere Praxis hinzutritt: die Praxis der Anerkennung. Erst in dem Moment, in welchem ein zweites Subjekt den Wert des vom ersten Subjekt produzierten Gegenstandes anerkennt, kann sich dieses sicher sein, dass das Selbstverständnis, welches es darin vergegenständlicht hat, auch soziale Existenz besitzt. Nur dann also, wenn Andere das Bild, das wir von uns selbst haben, bestätigen und ihm eine soziale Existenz verleihen, können wir eine gelungene Beziehung zu uns selbst aufbauen.
Fassen wir zusammen: Der Begriff des Selbstbewusstseins lässt sich für Hegel nur im Zusammenhang mit dem Begriff der Welt erläutern. In der Folge unterscheidet er dabei zwei Formen von Weltbeziehungen: die Praxis der Vergegenständlichung und die Praxis der Anerkennung. Zwar erlaubt erstere es dem Subjekt, sich selbst in der Welt gegenständlich zu werden und damit die Gestalt des Seins anzunehmen, jedoch erreicht diese Gestalt ihr volle Objektivität erst in jenem Moment, in dem sie von einem anderen Subjekt anerkannt wird. Erst dann kann sich das Subjekt sicher sein, dass die Überzeugungen, die es von sich hegt, wirklich geworden sind.

(ii) Freilich ist für Hegel mit dem Prinzip der Anerkennung die Entwicklungsgeschichte des Selbstbewusstseins noch nicht an ihr Ende gelangt. Vielmehr geht daraus erst jenes Theoriestück hervor, das ihm weit über die Philosophie hinaus zu Prominenz verholfen hat: das Verhältnis von Herrschaft und Knechtschaft. Der Entstehungsgrund dieses Verhältnisses ist, dass jedes Selbstbewusstsein seine Fähigkeit zur Selbstbestimmung durch ein jeweils anderes Selbstbewusstsein eingeschränkt sieht. Da sich das Tun des jeweils Anderen der eigenen Verfügungsgewalt entzieht und so der Selbstbestimmung Grenzen setzt, wird das andere Selbstbewusstsein zunächst als eine Bedrohung erfahren. In der Folge kommt es zu einem Konflikt zwischen den nach Anerkennung suchenden Selbstbewusstseinen: Ein jedes möchte in seiner absoluten Freiheit anerkannt werden und erwartet daher vom Anderen eine Unterwerfung unter seinen Willen. Da sich die Überzeugungen der beteiligten Selbstbewusstseine derart konträr gegenüberstehen, müssen sie in der Folge in einen Konflikt geraten, den Hegel nach und nach zu einem "Kampf auf Leben und Tod " (Hegel I 989: I 49) zuspitzt. Eine gewaltsame Form muss diese Auseinandersetzung für Hegel vor allem deshalb annehmen, weil die beteiligten Subjekte nur dadurch, dass sie ihr Leben für ihr Selbstverständnis aufs Spiel setzen, zeigen, dass sie wahrhaft freie Wesen sind. Erst dort, wo wir für unsere Vorstellungen von uns selbst eine existenzielle Gefahr in Kauf nehmen, so Hegel, zeigen wir, dass wir unsere tierische Existenz hinter uns gelassen haben und wahrhaft selbstbestimmte Wesen sind.

Entscheidend für Hegels Pathologiekonzeption ist nun, dass der Kampf um Anerkennung im ersten Anlauf scheitert. Sofern nämlich keines der beiden Subjekte dazu bereit ist, seine Überzeugungen aufzugeben, endet der Kampf mit dem Tod eines der Beteiligten. Was zunächst wie ein Sieg aussieht, ist auf den zweiten Blick aber eine Niederlage: das eigentliche Vorhaben, von einem anderen Subjekt Anerkennung zu erhalten, ist mit dem Tod des Anderen nämlich gescheitert. Aus eben diesem Scheitern lässt Hegels das überlebende Subjekt nun eine entscheidende Lehre ziehen: Es realisiert, »daß ihm das Leben so wesentlich als das reine Selbstbewußtsein ist « (ebd.: I 50). Anders gesagt: Anerkennung 
kann es nur im Leben geben und manchmal ist es nicht möglich, in diesem Leben genau jene Anerkennung zu erlangen, die man haben möchte. Mehr noch: Manchmal kann es besser sein, eine subordinierende Form der Anerkennung in Kauf zu nehmen, anstatt ganz ohne Anerkennung zu bleiben. Denn ohne Anerkennung droht der Rückfall in jenen Zustand des Mangels an Gewissheit, in welchem sich das Subjekt nicht sicher sein kann, ob es träumt oder wacht. Eine subordinierende Form der Anerkennung dagegen erlaubt es, Gewissheit von sich selbst zu erlangen, indem man sich den Überzeugungen, die Andere von einem hegen, unterwirft (Herrmann 201 2: 9 Iff.)

Im Fall des Selbstbewusstseins, dessen Entwicklungsgeschichte Hegel folgt, führt die eben skizzierte Einsicht nun dazu, dass sich das Subjekt beim nächsten Zusammentreffen mit einem anderen Subjekt dessen Überzeugungen unterwirft und sich selbst zum Knecht macht. Die Entstehung von Hegels berühmtem Herr-Knecht-Verhältnis ist also nicht einer gewaltsamen Bezwingung des Knechts geschuldet, sondern vielmehr einer freiwilligen Selbstunterwerfung: Es ist besser, eben jene subordinierende Anerkennung des Herrn in Kauf zu nehmen als ihn totzuschlagen und gar keine Anerkennung zu bekommen. Hegel führt uns also ein Verhältnis der freiwilligen Knechtschaft vor Augen, in welchem das Subjekt seine eigene Unterwerfung aufgrund seiner Abhängigkeit von Anerkennung in Kauf nimmt.

(iii) Hegels Überlegungen zur existentiellen Abhängigkeit von Anerkennung sind in der Sozialphilosophie des 20. Jahrhunderts verschiedentlich rezipiert und weitergeführt worden. Dabei war es vor allem die feministische Theoriebildung, für die sich Hegels Ansatz als besonders produktiv erwiesen hat. Exemplarisch zeigt sich das an Simone de Beauvoirs I949 erschienenem Klassiker Das andere Geschlecht, der die Geschlechterverhältnisse im Frankreich der I 94oer Jahre untersucht. Beauvoir stellt hier gleich zu Beginn die Frage: "Woher kommt die[] Unterwerfung bei der Frau? " (Beauvoir 2000: I4) Ihre Antwort auf diese Frage fällt ernüchternd aus: Zwar ist es für sie in erster Linie der Mann, der die Frau zum anderen seiner selbst macht, um ihr all jene Eigenschaften zuschreiben zu können, mit denen er sich selbst nicht identifizieren möchte. Allerdings würde ihm diese Unterwerfung der Frau als dem Anderen seiner selbst nicht gelingen, wenn diese nicht an ihrer eigenen Unterdrückung mitwirken würde. Die Unterdrückung der Frauen hat für Beauvoir daher zwei Quellen: Einerseits geht sie auf eine Unterwerfung durch Andere zurück, andererseits basiert sie auf einer freiwilligen Selbstunterwerfung.

Mit Selbstunterwerfung ist dabei gemeint, dass sich die Frau eben jene Ideale und Normen der Feminität zu eigen macht, welche ihre Unterdrückung begründen und strukturieren. Statt gegen die an sie herangetragen gesellschaftlichen Normen aufzubegehren und Widerstand zu leisten, werden sie von ihr affirmiert und verinnerlicht. Es ist eben dieses Phänomen der Selbstunterwerfung, das in der feministischen Theoriebildung bis heute immer wieder zum Gegenstand der Reflexion geworden ist, weil es deutlich macht, dass gesellschaftliche Ungleichheitsverhältnisse nicht einfach durch äußeren Zwang begründet werden, noch einfach auf der ideologischen Verblendung der sozialen Akteure beruhen. Vielmehr zeugt das Phänomen der Selbstunterwerfung von einem leidenschaftlichen Verhaftetsein an die Bedingungen der eigenen Unterwerfung (Butler 200I)

Beauvoir versucht das Phänomen der freiwilligen Selbstunterwerfung im Rückgriff auf Hegels Herr-Knecht-Verhältnis zu verstehen: Ganz so, wie dem Herrn der Knecht als das Unwesentliche gilt, gilt auch dem Mann die Frau als unwesentlich: Während er selbst das Universale und Allgemeine repräsentiert, schreibt er der Frau das Partikulare und Besondere zu: der Mann ist Mensch, die Frau ist Geschlecht. Ebenso wie der Knecht nun aufgrund seiner Abhängigkeit von Anerkennung seine unwesentliche Rolle akzeptiert, nimmt auch die Frau ihre unwesentliche Rolle an: "Die Frau erhebt nicht den Anspruch Subjekt zu sein, [...] weil sie ihre Bindung an den Mann als notwendig empfindet, ohne deren Reziprozität zu setzen, und weil sie sich oft in ihrer Rolle als Andere gefällt. " (Beauvoir 2000: I7) Zwar ist die Frau als das Andere des Mannes gesellschaftlich nicht im gleichen Maße wie dieser als Subjekt anerkannt, solange sie jedoch ihre Rolle z.B. als gute Hausfrau und Mutter ausfüllt, erhält sie zumindest eine partielle Form der Wertschätzung. Eben diese partielle Wertschätzung hat nun für Beauvoir zur Folge, dass sich die Frau mit ihrer gesellschaftlichen Stellung arrangiert: Während sie als Hausfrau und Mutter der Anerkennung durch den Mann sicher sein kann, droht sie als »Emanze « ihre gesellschaftliche Intelligibilität zu verlieren. Es ist gerade dieses mit dem Aufbegehren verbundene Risiko wie andersherum, jene mit der subordinierenden Anerkennung verbundene Sicherheit, die für Beauvoir dazu führt, dass sich die Frauen ihrer Generation mit ihrer untergeordneten Rolle arrangieren.

Beauvoir beschränkt sich nun nicht allein darauf, mit Hegel das Phänomen der Selbstunterwerfung für eine Analyse der gesellschaftlichen Situation der Frau im Frankreich der I 940er Jahre fruchtbar zu machen. Zugleich macht sie es sich zur Aufgabe, die Ursachen der Selbstunterwerfung neu zu durchdenken. Dabei geht sie davon aus, dass das Wesen des Menschen in der Freiheit besteht. Und zwar nicht deshalb, weil die Freiheit die wichtigste Eigenschaft des Menschen wäre, sondern vielmehr, weil der Mensch durch und durch Freiheit ist. Es ist das Wesen des menschlichen Bewusstseins selbst, sich im Zuge eines Weltentwurfs auf eine bestimmte Zukunft hin zu entwerfen und damit die natürliche gegebene Welt immer schon sinnhaft zu überschreiten und auszudeuten. 
Freiheit meint im Vokabular von Beauvoir entsprechend nicht Handlungsfreiheit, sondern die existenzielle Freiheit, das eigene Leben entwerfen zu können. Und diese Freiheit geht selbst da nicht verloren, wo einem Subjekt sämtliche Handlungsoptionen zu fehlen scheinen. Selbst in Gefangenschaft bleibt einem Subjekt immer noch die Freiheit, diese Situation nicht einfach hinzunehmen, sondern seinen Ausbruch zu entwerfen und zu versuchen (Sartre I993: 837). Obgleich nun also die existenzielle Freiheit nicht verloren zu gehen vermag, so kann sie doch verleugnet werden. Eben das, so Beauvoir, passiert in der Haltung der Unaufrichtigkeit. Hier versucht das Subjekt seine existenzielle Freiheit vor sich zu verbergen und sich selbst wie ein Ding zu behandeln, dessen Schicksal von außen festgelegt und vorherbestimmt ist. Warum aber sollte ein Subjekt seine Freiheit vor sich verbergen? Hier ist Beauvoirs Antwort deutlich: Die Freiheit wird vom Subjekt zunächst als Last erfahren. Beständig wird es von der "Qual der Wahl " heimgesucht und sieht sich dazu aufgefordert, seine Welt zu entwerfen. Die Unaufrichtigkeit erlöst von dieser existenziellen Überforderung, da sich das Subjekt vorgefertigten Rollenmustern zuwenden kann. Im Unterschied zu Hegel hat die Anerkennungsabhängigkeit ihre Ursache also nicht in einer existenziellen Not, sondern sie gründet vielmehr in einer existenziellen Last. Eben durch diese Verschiebung gelingt es Beauvoir das Phänomen der Selbstunterwerfung aus Hegels sehr speziellem gedanklichen Kontext zu lösen und für eine kritische Gesellschaftstheorie anschlussfähig zu machen.

\section{Marx: Die Einseitigkeit von Anerkennung}

Mit dem Namen Marx verknüpft man gemeinhin die Kritik der politischen Ökonomie, nicht jedoch eine Theorie der Anerkennung. Und dieses Urteil ist sicherlich richtig. Dennoch finden sich gerade beim jungen Marx eine Reihe von wichtigen Überlegungen zum Status intersubjektiver Anerkennung. Zentral ist dabei neben den Ökonomisch-philosophischen Manuskripten von I 844 auch das unmittelbar davor im Sommer entstandene "James Mill-Exzerpt «. Marx zeigt sich in beiden Texten konzeptionell noch wesentlich von Hegel beeinflusst, wenngleich er seine Überlegungen oftmals gerade in Abgrenzung zu ihm entwickelt werden. Anders als Hegel versteht er Arbeit und Anerkennung nämlich nicht mehr als zwei voneinander getrennte Realisierungsmöglichkeiten menschlicher Wesen, sondern als zwei intrinsisch aufeinander verwiesene Praktiken: Das Anerkennen bildet für ihn nicht das Andere der Arbeit, vielmehr ist Arbeit nur dann wirklich Arbeit, wenn sie zugleich auch eine Praxis der Anerkennung ist. Es ist eben dieser Umstand, auf den in jüngster Zeit eine ganze Reihe von Marx und Hegel-Interpreten aufmerksam gemacht haben (exemplarisch Brudney 2010). Damit haben sie nicht nur dazu beigetragen, eine Schieflage in der Marx-Rezeption zu korrigieren, insofern hier oftmals allein das Moment der Vergegenständlichung durch Arbeit als zentrales Moment von Marx' Überlegungen in den Blick geraten ist, sondern auch dazu, die Frage nach einer emanzipativen Ökonomie, in welcher Arbeit als menschengemachter Zusammenhang erscheint, wieder auf die sozialtheoretische Agenda zu setzen (Cohen 2009). Im Folgenden wird es mir darum gehen, kurz Marx' anerkennungstheoretische Überlegungen zu rekonstruieren (i), um im Anschluss daran die Pathologiediagnose vorzustellen, die Marx vor dem Hintergrund seines anerkennungstheoretisch unterfütterten Arbeitsbegriffs stellen kann (ii). Im dritten Schritt werde ich dann zeigen, wie heute an die von Marx gestellte Diagnose anzuknüpfen versucht wird (iii).

(i) An die Stelle, an der Rousseau auf den Begriff der Eigenliebe rekurriert und Hegel auf den Begriff des Selbstbewusstseins, tritt bei Marx der Begriff des Gattungswesens. Mit ihm will er systematisch jene Einsicht aufnehmen, die das Ergebnis von Hegels Überlegungen zum Kampf um Anerkennung dargestellt haben. Diese bestand ja gerade darin, dass das Selbstbewusstsein erkennen musste, dass ihm - in Hegels Worten -, "das Leben ebenso wesentlich als das reine Selbstbewußtsein ist « (Hegel I 989: I 50). Es ist eben diese Einheit von Leben und Selbstbewusstsein, die Marx im Begriff des Gattungswesens vereinigt, wenn er den Mensch einerseits als ein Naturwesen bestimmt, das produziert und konsumiert, um seine Lebensbedürfnisse zu stillen, und andererseits als ein Freiheitswesen, das sich selbst seine Zwecke und Zielsetzungen gibt (Marx 2009: 89). Nun scheinen beide Motive zunächst in zwei ganz unterschiedliche Richtungen zu verweisen: Dort das Reich der Notwendigkeit und hier das Reich der Freiheit. Die Pointe von Marx' Gattungsbegriff besteht nun aber genau darin, beide Motive im Begriff des Gattungswesens so miteinander zu amalgamieren, dass er dieses nur dann verwirklicht sehen kann, wenn ein Individuum frei und selbstbestimmt für seine Bedürfnisse produziert. Erst dort, wo das Reich der Notwendigkeit und das Reich der Freiheit miteinander versöhnt sind, ist das menschliche Gattungswesen für Marx realisiert. Freilich nun kann eine solche Versöhnung für Marx nicht mehr durch solitäre Produktion geleistet werden. Die gewachsenen und wachsenden Bedürfnisse der Individuen lassen sich nur im gesellschaftlichen Austausch befriedigen, was zur Folge hat, dass die Menschen ihr Gattungswesen letztlich nur kooperativ verwirklichen können. Dafür ist es einerseits notwendig, dass die Individuen ihre eigenen Bedürfnisse und die Bedürfnisse von Anderen kennen und anerkennen - denn nur so können sie ihr Naturwesen verwirkllichen -, und andererseits ist es notwendig, dass sie ihre Bedürfnisbefriedigung gemeinschaftlich in einem selbstbestimmten Prozess organisieren - denn nur so können sie ihren Austausch als Ausdruck der Freiheit verstehen. 
Vor eben diesem Hintergrund formuliert Marx gegen Ende seines so genannten »Mill-Exzerptes « in wenigen Sätzen die Vision einer kommunistischen Produktionsweise. In ihr, so hält er fest, hätten die Individuen "sich selbst und den anderen doppelt bejaht " (ebd.: 206). Ausgehend von dieser doppelten Bejahung unterscheidet Marx im folgenden Absatz denn auch zwischen vier Beziehungen, die in der kommunistischen Produktionsweise am Werk sein sollen: Erstens, so Marx, würde ein Individuum A in der Produktion eines Gegenstandes seine Individualität vergegenständlichen und sich dadurch als Einzelwesen in seinen körperlichen und technischen Fähigkeiten bestätigt wissen. Zweitens würde Individuum A durch die Herstellung dieses Gegenstandes zugleich ein Bedürfnis von Individuum B befriedigen und dadurch dieses in seiner Individualität bestätigen. Drittens würde B im Konsum des Gegenstandes P nicht nur unmittelbar das Produkt genießen, sondern zugleich auch die unterliegende soziale Beziehung S. Er würde genießen, dass A den Gegenstand $P$ aus freien Stücken für sein Bedürfnis produziert hat. Viertens würde $A$ genießen, dass $B$ in seiner Konsumtion ihre soziale Beziehung $S$ als Ausdruck ihrer gemeinsamen Freiheit und ihrer wechselseitigen Bezogenheit genießt. Würden mit der kommunistischen Produktionsweise alle diese vier Aspekte der Bejahung verwirklicht, dann wäre das menschliche Gattungswesen hier vollständig realisiert: A und B würden sich nämlich durch ihren unmittelbaren Genuss an Produktion und Konsumtion einerseits als Naturwesen bejahen, wie sie sich anders herum durch ihren unmittelbaren Genuss an der Art und Weise, wie sie das Verhältnis der Produktion gestaltet haben, als Freiheitswesen bejahen würden.

Wenn Marx nun als Zusammenfassung der eben eingeführten vier Formen der Bejahung festhält: "Unsere Productionen wären eben so viele Spiegel, woraus unser Wesen sich entgegen leuchtete " (ebd.: 207), dann lässt sich daran deutlich machen, dass es in der kommunistischen Produktionsweise nicht die Produkte selbst, sondern die mit ihnen verbundenen Einstellungen sind, welche für die Realisierung des Gattungswesens verantwortlich sind. Letzteres ist nämlich nicht allein dadurch möglich, dass Gegenstände produziert werden, die irgendein Bedürfnis befriedigen. Dicses Kriterium würde nämlich auch das marktvermittelte Tauschsystem des Kapitalismus erfüllen. Für die kommunistische Produktionsweise gilt vielmehr, dass die Befriedigung des Bedürfnisses eines Anderen Teil der Befriedigung des eigenen Bedürfnisses ist. Der Genuss des Produktes P durch B soll für A ein Genuss sein. Es ist eben diese übergreifende Struktur des kommunistischen Genießens, welche die wahrhaft menschliche Produktionsweise auszeichnen soll. Noch deutlicher wird das auf der Ebene der Freiheit: Auch hier soll der Genuss der sozialen Beziehung $\mathrm{S}$ durch B die Voraussetzung für den Genuss von A sein. Genossen wird also von jedem Akteur doppelt: Einerseits genießt er das eigene individuelle Tun, andererseits genießt er das dadurch hervorgebrachte
Genießen des Anderen. Was den an der kommunistischen Produktionsweise beteiligten Mitgliedern also aus den Produktionen entgegenleuchtet, ist daher nicht irgendeine spezifische Qualität des Produktes, sondern vielmehr ihre wechselseitige, freie Bezogenheit aufeinander, die sich in diesem Produkt vergegenständlicht.

Unter der Hand führt Marx also eine dritte Dimension des Begriffs des Gattungswesens ein: Der Mensch ist nicht nur Naturwesen und Freiheitswesen, sondern auch Gesellschaftswesen, weil der andere Mensch dem Menschen ein Genuss ist. Hinter Marx' vierfacher Form der Bejahung verbirgt sich daher das Bild einer Sozialordnung, in welcher sich die Beteiligten nur gemeinsam realisieren können. Diese wechselseitige Angewiesenheit aufeinander wird von den sozialen Akteuren aber nicht als ein äußerer Zwang empfunden, sondern vielmehr als Voraussetzung der Realisierung ihrer individuellen Zwecke. Nur in Resonanzbezügen mit Anderen können sie sich voll und ganz verwirklichen. Die damit einhergehende Bedeutung der Gemeinschaft für das menschliche Leben hat Daniel Brudney mit Hilfe der Unterscheidung zwischen übergreifenden und ineinandergreifenden Zielen auf den Punkt gebracht (Brudney 20I0): Im ersten Fall verfolgen Individuen gemeinsam geteilte Ziele, weil sie diese Ziele nur mit vereinten Kräften erreichen können. Es sind daher gleichsam instrumentelle Gründe, aus denen heraus die Individuen kooperieren. Im Unterschied dazu ist bei der Verfolgung ineinandergreifender Ziele die Kooperation selbst der Zweck des Handelns. Die Subjekte sind hier nicht mehr nur miteinander, sondern füreinander tätig, weil das Erreichen der Ziele des Anderen intrinsisch mit dem Erreichen ihrer eigenen Ziele verknüpft ist. Es ist eben dieser Gedanke, der auch Marx vor Augen gestanden haben muss, als er festhielt: "Das menschliche Wesen ist erst für den gesellschaftlichen Menschen; denn erst hier ist sie für ihn da als Band mit dem Menschen, als Dasein seiner für den andern und des andern für ihn." (Marx 2009: I I 8)

(ii) Vor dem Hintergrund von Marx' Skizze einer kommunistischen Produktionsweise, lässt sich nun in einem zweiten Schritt die von ihm diagnostizierte Pathologie des Kapitalismus deutlich machen. In diesem produzieren die Individuen nämlich nicht mehr füreinander, sondern nur noch miteinander. Anlass der eigenen Produktion ist hier nicht mehr das unmittelbare Interesse an den Bedürfnissen anderer, sondern allein das Interesse am Erwerb materieller Güter. In der kapitalistischen Gesellschaft, so Marx ironisch in Bezug auf Adam Smith, ist jeder nur ein "Kaufmann « für den anderen (Marx 2009: I 96). Was damit gemeint ist, zeigt sich paradigmatisch an der Vermittlung des Warenverkehrs durch das Geldmedium. Der geldvermittelte Tausch geht mit einer sozialen Überbrückungsleistung einher, die es den Individuen erlaubt, sich persönlich soweit voneinander zu distanzieren, dass sie sich wechselseitig 
nur noch als anonyme Tauschpartner begegnen. Eben dadurch aber werden sie wechselseitig blind füreinander. In dem Moment nämlich, wo die Bedürfnisse von Anderen nur noch Mittel zum Zweck des materiellen Gütererwerbs sind, geht jedes Verständnis für den Genuss des Genießens des Anderen verloren. Der gedankliche Übergang von der kommunistischen zur kapitalistischen Produktionsweise lässt sich daher wesentlich an einer Veränderung im Selbstverständnis der Akteure festmachen: Statt sich als gesellschaftliche und mit anderen verbundene Wesen zu sehen, begreifen sich die Einzelnen als solitäre, von anderen getrennte Wesen. Es ist eben dieser begriffliche Übergang von einer holistischen zu einer atomistischen Gesellschaftskonzeption, welche für Marx die grundlegende Deformation des Kapitalismus ausmacht. Er kann daher festhalten, dass im Kapitalismus »das Wesensband, das den Menschen an andere knüpft, als unwesentliches Band erscheint und vielmehr die Trennung vom anderen Menschen als wahres Dasein. « (Ebd.: I 96)

Als Ursache der atomistischen Gesellschaftskonzeption des Kapitalismus macht Marx vier Formen der Entfremdung aus. Zunächst die Entfremdung des Arbeiters von seinem Produkt: Weil er als Lohnarbeiter keine Verfügungsmacht über die von ihm hergestellten Produkte hat, treten ihm diese als fremde gegenüber. Eben dadurch verlieren sie aber auch die Möglichkeit, das Naturwesen des Arbeiters widerzuspiegeln. Eng Verbunden mit der Entfremdung vom Produkt ist die zweite Form der Entfremdung, die sich auf den Herstellungsprozess bezieht. Insofern der Arbeiter als Lohnarbeiter fremdbestimmten Routinen unterliegt, kann es sich nicht als Freiheitswesen verwirklichen. Als drittes Moment führt Marx dann die Entfremdung vom anderen Menschen an: Weil im kapitalistischen Warentausch die positive Beziehung auf die Bedürfnisse von Anderen abhanden gekommen ist, unterhalten die sozialen Akteure hier nur noch versachlichte soziale Beziehungen zueinander. Marx spricht diesbezüglich auch davon, dass wir nur noch die Sprache der Gegenstände miteinander reden. Problematisch an dieser Sprache ist, dass die individuelle Bedürftigkeit in ihr nur als ausbeutbare Schwäche in Erscheinung zu treten vermag, welche den marktwirtschaftlich handelnden Akteuren eine Chance zur Ausdehnung ihrer ökonomischen Macht gibt

Wenn Marx nun als vierten Punkt die Entfremdung vom Gattungswesen anführt, so scheint es, dass er hier eigentlich nur die drei bisher genannten Formen der Entfremdung noch einmal zusammenfasst. Mit der Entfremdung des Menschen als Naturwesen, als Freiheitswesen und als Gesellschaftswesen hat Marx ja bereits eben jene drei Momente zur Sprache gebracht, von denen ich gezeigt habe, dass sie den normativen Kern seines Gattungswesensbegriffs ausmachen. Was Marx dem nun als vierte Form der Entfremdung hinzufügt, ist, dass den Menschen die drei zuerst genannten Entfremdungsformen nicht als Entfremdungsformen vor Augen treten. Mehr noch: Die kapitalistische Produktionsweise wird nicht als Verfallsform, sondern als Realisierung der Natur des Menschen verstanden. Genau damit stoßen wir nun aber zum eigentlichen Kern von Marx' Kritik der politischen Ökonomie vor: Der Kapitalismus etabliert eine gesellschaftliche Selbstdeutung, in welcher die Einzelnen ihren entfremdeten Zustand gleichsam als naturgegeben begreifen. Ursache hierfür ist - so wird Marx später im Kapital im seinen berühmten Kapitel über den Fetischcharakter der Ware zeigen -, dass die sozialen Verhältnisse von Ware, Geld und Kapital den Einzelnen als naturwüchsige Mächte gegenübertreten, weshalb ihnen ihre Rollen als Warenverkäufer, Vertragspartner und Eigentümer selbstverständlich scheinen. Gestützt wird dieser falsche Schein für Marx noch durch das Recht, insofern dieses die Einzelnen gerade nicht positiv als Gattungswesen, sondern vielmehr negativ als Individualwesen aufeinander bezieht, da es das Kennzeichen liberaler Freiheitsrechte ist, den Einzelnen vor der Einflussnahme von Anderen zu schützen (Schmidt am Busch 20I I: I 2off.). Das Recht erlaubt es so, den Hobbesschen Naturzustand eines Kampfes aller gegen alle in den Rechtszustand hinein zu verlängern. Nur wird der Kampf nun nicht mehr mit den Mitteln der physischen Gewalt, sondern mit Hilfe der zum Tausch angebotenen Waren geführt: „Unsere wechselseitige Anerkennung über die wechselseitige Macht unserer Gegenstände ist aber ein Kampf, und im Kampf siegt, wer mehr Energie, Kraft, Einsicht oder Gewandtheit besitzt. « (Ebd.: 204, dazu: Quante 2009: 292) In das Zentrum von Marx Analyse tritt so eine doppelte Pathologie: Einerseits führt die kapitalistische Produktionsweise zu einer gesellschaftlichen Fehldeu tung der Akteure, in der diese ihren Gattungscharakter vergessen und sich nur noch als Individualwesen verstehen - eine Pathologie, die wir im Anschluss an Marx als Anerkennungsvergessenheit bezeichnen können. Andererseits führt genau diese Anerkennungsvergessenheit zu einer Fehlaneignung bestehender Anerkennungspraktiken: So wird das Recht unter kapitalistischen Bedingungen nicht länger als Schutzraum vor dem Übergriff von Anderen verstanden, sondern vielmehr ist es ein Mittel für die "Absicht der Plünderung " (Marx 2009: 204) - eine Pathologie, die wir als Anerkennungseinseitigkeit bezeichnen können. Eben jenes letzte Moment ist es, das in Anknüpfung an Marx zum Gegenstand weiterführender sozialtheoretischer Überlegungen geworden ist.

(iii) Marx' anerkennungstheoretische Überlegungen sind in jüngster Zeit von unterschiedlichen Seiten aufgegriffen worden. Jüngst etwa hat Axel Honneth zu zeigen versucht, dass die seiner Anerkennungstheorie zu Grunde liegende Idee der sozialen Freiheit eben jener Idee von Freiheit entspricht, die Marx ausgehend von seiner Konzeption einer kommunistischen Produktionsweise zu fassen versucht hat (Honneth 20I 5). Obwohl Honneth Marx dabei für seine konzeptionelle Verengung auf die Sphäre des marktvermittelten Tausches kritisiert und darin 
eine der Hauptursachen des Scheiterns der sozialistischen Bewegung sieht, besteht ein Anliegen seiner Anerkennungstheorie darin, danach zu fragen, unter welchen Bedingungen der Markt zu einer Sphäre der sozialen Freiheit werden kann (Honneth 20I I: 3 I fff). Mit Marx teilt er daher zunächst einmal den normativen Ausgangspunkt, demzufolge die Beziehung zu Anderen als Bedingung der Möglichkeit der eigenen Selbstverwirklichung gesehen werden muss. Vor dem Hintergrund dieser Prämissen ist es nun wenig verwunderlich, dass Honneth auch die Pathologiediagnose der Anerkennungsvergessenheit der Sache nach mit Marx teilt. Auch für ihn führt nämlich das Vergessen der wechselseitigen sozialen Bezogenheit zu einer verzerrten gesellschaftlichen Selbstauslegung, in der sich die sozialen Akteure indifferent gegeneinander verhalten.

Bevor Honneth zu dieser Pathologiediagnose kommt, geht es ihm - und hier unterscheidet er sich von Marx - zunächst einmal darum, den Daseinsgrund der rechtlichen Anerkennung herauszupräparieren. Der Sinn des positiven Rechts besteht für ihn darin, den Individuen einen Spielraum zuzuerkennen, in welchem sie nach beliebigen selbstgesetzten Zwecken handeln können. Die Gewährung einer solchen negativen Freiheit, die sich in erster Linie als Abwesenheit von äußeren Hindernissen bei der Verwirklichung von subjektiven Handlungszielen bestimmt, hat für Honneth nun einen ethischen Sinn: Dieser besteht darin, den Individuen einen Schutzraum zu gewähren, innerhalb dessen sie ihre eigenen Interessen, Vorlieben und Präferenzen erkunden und formen können. Die rechtliche Anerkennung sichert dem Individuum so einen experimentellen Raum der ethischen Selbstverständigung (Honneth 20 I2: I 32 f.). In diesem Selbstverständigungsprozess spielt die individuelle Verfügungsmacht über Gegenstände eine zentrale Rolle, weil sich an ihnen die jeweils eigenen Selbstentwürfe vergegenständlichen und reflektieren lassen. Erst an den Gegenständen erfährt die allgemeine Existenz der Rechtsperson daher ihre Konkretisierung und Verwirklichung, weshalb das Recht auf privates Eigentum für Honneth als ein notwendiger Bestandteil des positiven Rechts gilt. Seinen ethischen Zweck kann das Recht nun allein unter der Bedingung verwirklichen, dass sich die Individuen in ihrem wechselseitigen Umgang miteinander einen eben solchen Raum der experimentellen Selbstfindung zugestehen. Das heißt, dass sie sich und Anderen das Recht zuerkennen müssen, Handlungen ohne öffentlichen Rechtfertigungsdruck auszuüben, was nur dadurch möglich ist, dass sie von allen ethischen Beweggründen und persönlichen Motivlagen absehen (ebd.: I 48f). Die Einnahme der Rolle der Rechtsperson setzt daher die Fähigkeit voraus, in der Interaktion mit Anderen von individuellen Überzeugungen abstrahieren und die eigenen ethischen Grundsätze einklammern zu können. Honneth macht damit deutlich, dass die trennende Funktion des Rechts eigentlich einen ethischen Sinn hat: Sie soll es den Individuen erlauben, sich hinter die Handlungserwartungen ihrer
Mitmenschen zurückzuziehen und alternative Weisen des ethischen Handelns erproben zu können.

Entscheidend ist für Honneth nun, dass das Recht seinen ethischen Sinn nur unzureichend zu erfüllen vermag, weil der durch es zugesprochene Schutzraum wesentlich monologisch konzipiert ist. Der Rückzug hinter den Schutzwall des Rechts hat für den Einzelnen die Funktion, sich aus sozialen Begründungspflichten zu lösen, um ausschließlich mit sich allein auszumachen, was das Richtige oder Gute ist. Entscheidend ist nun aber, dass in einer solchen monologischen Perspektive ethische Selbstverständigung letztlich gerade nicht möglich ist. Diese setzt nämlich mindestens ein virtuelles Gespräch mit sich selbst voraus, in dem wir uns vor unserem imaginären Gesprächspartner rechtfertigen müssen (ebd.: I 52 f.). Insofern daher jeder Entwurf eines guten Lebens notwendig mit einer Form der intersubjektiven Verständigung einhergeht, ist die Rolle der Rechtsperson gerade nicht dazu geeignet, einen ethischen Standpunkt zu entwickeln. Der eigentliche Zweck des subjektiven Rechts ist es daher, temporär einen Rückzug aus ethischen Beratschlagungsprozessen zu ermöglichen, der dann für eine individuelle Selbstverständigung und Rückversicherung der eigenen Position genutzt werden kann. Wo diese handlungsunterbrechende Funktion des Rechts verabsolutiert wird, kommt es für Honneth zu sozialen Pathologien. Das ist etwa überall dort der Fall, wo sich Individuen im Fall von sozialen Streitigkeiten wechselseitig auf ihre Rolle als Rechtsträger versteifen und alle kommunikativen Schlichtungsangebote ausschlagen oder dort, wo die entlastende Funktion des Rechts zum alleinigen Bezugspunkt des eigenen Selbstverständnisses wird (ebd.: 160). In beiden Fällen besteht die soziale Pathologie darin, dass die durch die rechtliche Anerkennung ermöglichten Handlungsspielräume nicht mehr angemessen verstanden werden. Statt die rechtliche Anerkennung als temporären Rückzugsraum aus sozialen Beziehungen zu nutzen, werden diese nach dem Muster der rechtlichen Anerkennung gelebt. Die Individuen verlieren so nicht nur jeglichen Sinn für sozial angemessene Kommunikationsmuster, sondern darüber hinaus auch das Bewusstsein dafür, dass sie für ihre Selbstverwirklichung auf ihre wechselseitige positive Bezugnahme angewiesen sind. Während Marx die Ursachen der Fehlaneignung des Rechts auf die kapitalistische Produktionsweise zurückgeführt hat, macht uns Honneth damit deutlich, dass diese Fehlaneignung von einer im subjektiven Recht selbst angelegten Widersprüchlichkeit herrührt: Eigentlich als Schutzwall für die ethische Selbstverständigung gedacht, vermag es diese Rolle aufgrund seiner trennenden Funktion gerade nicht auszuüben. Eben deshalb können die durch das Recht zugesicherten Freiheitsspielräume stets nur den Charakter temporärer Unterbrechungen haben, die gleichsam vorbereitend der Wiederaufnahme der Verständigung mit Anderen dienen. Wo dieser Charakter rechtlicher Anerkennung von den sozialen 
Akteuren nicht mehr verstanden wird, kann es zur Pathologie der Anerkennungseinseitigkeit kommen.

\section{Drei Pathologien der Anerkennung}

Im Durchgang durch die Überlegungen von Rousseau, Hegel und Marx ist deutlich geworden, dass alle drei nicht nur genuine Beiträge zu einer Theorie der Anerkennung geliefert, sondern jeweils auch ganz eigene Vorstellungen von Pathologien der Anerkennung entwickelt haben. Der normative Horizont der wechselseitigen Anerkennung - sei er durch das Konzept der Eigenliebe, den Begriff des Selbstbewusstseins oder die Idee des Gattungswesens motiviert - dient allen drei Autoren dazu, eine spezifische Pathologie zu identifizieren: Bei Rousseau die Idee der Anerkennungsbesessenheit, bei Hegel die Idee der Anerkennungsabhängigkeit und bei Marx die Idee der Anerkennungsvergessenheit. Alle drei Pathologiediagnosen, so habe ich im Ausgang von den Überlegungen von Hartmut Rosa, Simone de Beauvoir und Axel Honneth gezeigt, haben auch heute nicht an ihrer Aktualität verloren, sondern lassen sich unter modifizierten Bedingungen nach wie vor für eine kritische Gesellschaftstheorie fruchtbar machen.

Ich will nun abschließend noch kurz darauf eingehen, in welchem Verhältnis die jeweiligen Pathologien der Anerkennung stehen. Dafür ist es hilfreich, auf die in jüngerer Zeit immer wieder herangezogene Unterscheidung zwischen Pathologien erster Ordnung und Pathologien zweiter Ordnung zurückzugreifen (Celikates 2009). Unter ersterer sollen dabei all jene gesellschaftlichen Missstände gefasst werden, die ihren Ursprung in gesellschaftlichen Verhältnissen haben. Dort wo subjektives Leid systematisch durch die sozialen Bedingungen hervorgebracht wird, haben wir es mit einer Pathologie erster Ordnung zu tun. Im Gegensatz dazu meinen Pathologien zweiter Ordnung all jene gesellschaftlichen Missstände, die es den sozialen Akteuren verunmöglichen, kritisch auf die Missstände erster Ordnung Bezug zu nehmen. Dort also, wo Unrechtsverhältnisse von den sozialen Akteuren gar nicht als Unrechtsverhältnisse wahrgenommen werden, sondern als natürlich gegeben hingenommen werden, haben wir es mit Pathologien zweiter Ordnung zu tun. Vor diesem Hintergrund ist es nun möglich, die von Rousseau, Hegel und Marx diagnostizierten Pathologien zueinander ins Verhältnis zu setzen:

Rousseaus Überlegungen zur Anerkennungsbesessenheit haben uns gezeigt, dass Anerkennung die Ursache der Entstehung von Ungleichheitsverhältnissen sein kann. Das hat seinen Grund darin, dass das Anerkennen stets relational verfasst ist. Jemanden wertzuschätzen bedeutet für Rousseau, ihn ins Verhältnis zu Anderen zu setzen. Anerkennung und soziale Hierarchisierung sind daher aufs engste miteinander verbunden. $\mathrm{Zu}$ einer Pathologie wird dieser Umstand freilich erst in dem Moment, wo bestimmten gesellschaftlichen Gruppen aufgrund ihres Geschlechts, ihrer sozialen Herkunft oder ihrer Nationalität systematisch Anerkennung vorenthalten wird. Rousseaus Pointe besteht daher darin zu zeigen, dass die Praxis der Anerkennung eine große Anschlussfähigkeit für die Etablierung von Pathologien erster Ordnung besitzt.

Marx' Überlegungen zur Anerkennungsvergessenheit ergänzen diese Diagnose nun von der anderen Seite: Sie zeigen uns nämlich, dass diese Pathologien von den sozialen Akteuren in dem Moment nicht mehr als Pathologien wahrgenommen werden, wo sie ihre wechselseitige Bezogenheit nicht mehr in Begriffen der Anerkennung zu deuten vermögen, weil sie durch die gesellschaftlichen Verhältnisse in eine Beziehung zueinander gesetzt wurden, in der sie sich wechselseitig nur noch als Mittel zum Zweck ihrer eigenen Bedürfnisbefriedigung wahrnehmen. Es ist dieser Einschluss in ein falsches Selbstverständnis, der es verhindert, dass die sozialen Akteure defizitäre Anerkennungsverhältnisse als solche wahrzunehmen und zu thematisieren verstehen. Die Pointe von Marx' Ansatz besteht also darin, die mit dem kapitalistischen Warenverkehr verbundene rechtliche Anerkennung als eine soziale Praxis zu verstehen, welche die gesellschaftlichen Selbstdeutungen der Individuen systematisch verzerrt. Seine Idee der Anerkennungsvergessenheit bringt damit paradigmatisch eine Pathologie zweiter Ordnung zum Ausdruck.

Hegels Konzept der Anerkennungsabhängigkeit schließlich scheint mir das bisher in Anschlag gebrachte Deutungsschema zu erweitern: Zwar stellt das Phänomen der Anerkennungsabhängigkeit eine Pathologie zweiter Ordnung dar, insofern es erklären soll, warum die von sozialer Ungleichheit Betroffenen nicht als Unrechtsverhältnisse wahrgenommen werden. Im Gegensatz zu Marx wird die Ursache dieser Haltung jedoch nicht mit einem strukturell versursachten Reflexionsdefizit der sozialen Akteure begründet, sondern mit existenziellen Gründen: Die von subordinierender Anerkennung Betroffenen begehren für Hegel nicht deswegen nicht gegen die sozialen Verhältnisse auf, weil sie kein Unrechtsbewusstsein für diese hätten. Vielmehr nehmen sie soziale Ungleichheitsverhältnisse bewusst in Kauf, weil sie diese für die einzige Möglichkeit halten, überhaupt gesellschaftliche Anerkennung zu erhalten. Hegel zeigt uns damit, dass nicht nur die Vergessenheit von Anerkennung, sondern gerade auch ihre Dringlichkeit zur Ursache von sozialer Ungleichheit werden kann.

Fassen wir die vorangegangenen Überlegungen zusammen, dann lässt sich die Aufgabe einer kritischen Gesellschaftstheorie nach Rousseau, Hegel und Marx so verstehen, dass es ihre Aufgabe ist, die Praxis des Anerkennens selbst auf ihre hierarchisierenden und subordinierenden Effekte zu befragen und das Bewusstsein dafür wachzuhalten, dass 
Anerkennung für soziale Akteure nicht nur ein Mittel der Selbstverwirklichung, sondern auch der Unterwerfung sein kann. Dieser Aufgabe kann sie auf zwei Weisen gerecht werden: Einerseits muss sie das Entstehen und das Bestehen von Pathologien der Anerkennung erster Ordnung freilegen, indem sie zeigt, wie das Begehren von Anerkennung zur Etablierung von ungerechtfertigten sozialen Ungleichheitsverhältnissen führen kann. Andererseits muss sie Pathologien der Anerkennung zweiter Ordnung dadurch freilegen, dass sie Einseitigkeiten in der gesellschaftlichen Selbstverständigung aufdeckt und versucht, alternative, nicht-subordinierende Ancrkennungsressourcen zu erschließen und zu stärken.

\section{Literatur}

Brudney, Daniel (2010): "Gemeinschaft als Ergänzung“, in: Deutsche Zeitschrift für Philosophie Bd. 58, Heft 2, I95-2 I9.

Butler, Judith (200I): Psyche der Macht. Das Subjekt der Unterwerfung, Frankfurt am Main: Suhrkamp.

Castell, Robert (2000): Die Metamorphosen der sozialen Frage. Eine Chronik der Lohnarbeit, Kontanz: UVK.

Celikates, Robin (2009): Kritik als soziale Praxis. Gesellschaftliche Selhstverständigung und kritische Theorie, Frankfurt am Main: Campus.

Cohen, Gerald (2009): Sozialismus. Warum nicht?, München: Knaus.

De Beauvoir, Simone (2000): Das andere Geschlecht. Sitte und Sexus der Frau, Reinbek bei Hamburg: Rowohlt.

Dent, N. J. H. (I 988): Rousseau. An Introduction to his Psychological, Social and Political Theory, Oxford: Basil Blackwell.

Donzelot, Jacques (1984): L'invention du social. Essai sur le déclin des passions politiques, Paris: Fayard.

Hegel, G. W. F. (1989): Phänomenologie des Geistes, Werke Bd. 3, Frankfurt am Main: Suhrkamp.

Herrmann, Steffen (201 2): Symbolische Verletzharkeit. Die doppelte Asymmetrie des Sozialen nach Hegel und Levinas, Bielefeld: transcript.

Honneth, Axel (20 I I): Das Recht der Freiheit. Grundriß einer demokratischen Sittlichkeit, Berlin: Suhrkamp.

- (2015): Die Idee des Sozialismus. Versucht einer Aktualisierung, Berlin Suhrkamp.

Kojève, Alexandre (1 975): Hegel. Eine Vergegenwärtigung seines Denkens, Frankfurt am Main: Suhrkamp.

Kuch, Hannes (2012): Herr und Knecht. Anerkennung und symbolische Macht im Anschluss an Hegel, Frankfurt am Main: Campus.

Marx, Karl (2009): Ökonomisch-philosophische Manuskripte, Frankfurt am Main: Suhrkamp.
Neuhouser, Frederick (2008): "Rousseau und das menschliche Verlangen nach Anerkennung ", in: Deutsche Zeitschrift für Philosophie, Bd. 56, Heft 6, 899-922.

- (201 2): Pathologien der Selhstliebe. Freibeit und Anerkennung bei Rousseau, Berlin: Suhrkamp.

- (20 I 4): Rousseaus's Critique of Inequality. Reonstructing the Second Discourse, Cambridge: University Press.

Quante, Michael (2009): "Kommentar«, in: Marx, Karl, Ökonomisch-philosophische Manuskripte, Frankfurt am Main: Suhrkamp.

Rosa, Hartmut (2013): Beschleunigung und Entfremdung. Entwurf einer Kritischen Theorie spätmoderner Zeitlichkeit, Berlin: Suhrkamp.

- (201 2): Welthezichungen im Zeitalter der Beschleunigung. Umrisse einer neuen Gesellschaftskritik, Berlin: Suhrkamp.

Rousseau, J.-J. ( 1998 ): Emil oder üher die Erzichung, Paderborn: Schöningh.

- (2008): Diskurs üher die Ungleichheit, übersetzt von Heinrich Meier, Paderborn: Schöningh.

Sartre, Jean-Paul ( I 993): Das Sein und das Nichts. Versuch einer phänomenologischen Ontologie, Reinbek bei Hamburg: Rowohlt.

Schmidt am Busch, Hans Christoph (20 I I): „Anerkennung« als Prinzip der Kritischen Theorie, Berlin: de Gruyter.

Taylor, Charles ( I 996): Quellen des Selbst. Die Entstehung der neuzeitlichen Identität, Frankfurt am Main: Suhrkamp. 


\title{
Warum Kritik?
}

Begründungsformen kritischer Theorien

Herausgegeben von

Sven Ellmers und Philip Hogh

\author{
VELBRÜCK \\ WISSENSCHAFT
}




\section{Inhaltsverzeichnis}

SVEN Ellamers Uni) PhII.IP Hogh

Warum Kritik? Zur Einleitung

PHII.IP HoGH

Apathie Kälte Verdinglichung. Zur gesellschaftlichen Wirklichkeit moralischer Indifferenz . . . . . . . . . . . . .

CHRISTOPH HENNING

Perfektionismus als kritische Theorie. Bemerkungen zur Selbstentfaltung als Kategorie sui generis. ............

JOCHEN GIMMEI

Ist Kritik erlaubt? - Zu einigen konzeptionellen Schwierigkeiten des Begriffs Kritik bei Kant . . . . . . . . . . . . . .

HAUKF: BRUNKHORST

Touching the Void - Kritische Theorie als dialektischer Negativismus. Thesen zur Hegelschen Logik . . .

HANNES KUCH

Ökonomie, Subjektivität und Sittlichkeit. Hegel und die Kritik des kapitalistischen Marktes . . . . . . . . . ....

Erste Auflage 20I7

(C) Velbrück Wissenschaft, Weilerswist 2017 www.velbrueck-wissenschaft.de

Printed in Germany

ISBN 978-3-95832-063-5

Bibliografische Information der Deutschen Nationalbibliothek Die Deutsche Nationalbibliothek verzeichnet diese Publikation in der Deutschen Nationalbibliografie; detaillierte bibliografische Daten sind in Internet über http://dnb.ddb.de abrufbar.

STFHFiN K. HFRRMANN

Drei Pathologien der Anerkennung.

Grundlagen einer kritischen Gesellschaftstheorie

nach Rousseau, Hegel und Marx . . . . . . . . . . . . . . . .

FRANK KUHN

Moral im »Kapital «? - Hat Marx' Kritik der politischen

Ökonomie normative Grundlagen? . . . . . . . . .

CHRISTINE KIRCHHOFF

Kultur und Illusion. Die Begründung von Kritik mit der Psychoanalyse Sigmund Freuds . . . . . . . . .

FABIAN FREYENHAGEN

"Aber was das Unmenschliche ist, das wissen wir sehr genau «

Zur Normativitätsproblematik bei Adorno ........... 